\title{
Research Article \\ Symmetric Three-Term Recurrence Equations and Their Symplectic Structure
}

\author{
Roman Šimon Hilscher ${ }^{1}$ and Vera Zeidan ${ }^{2}$ \\ ${ }^{1}$ Department of Mathematics and Statistics, Faculty of Science, Masaryk University, Kotlárská 2, \\ 61137 Brno, Czech Republic \\ ${ }^{2}$ Department of Mathematics, Michigan State University, East Lansing, MI 48824-1027, USA
}

Correspondence should be addressed to Roman Šimon Hilscher, hilscher@math.muni.cz

Received 11 March 2010; Accepted 1 May 2010

Academic Editor: Martin Bohner

Copyright @ 2010 R. Šimon Hilscher and V. Zeidan. This is an open access article distributed under the Creative Commons Attribution License, which permits unrestricted use, distribution, and reproduction in any medium, provided the original work is properly cited.

We revive the study of the symmetric three-term recurrence equations. Our main result shows that these equations have a natural symplectic structure, that is, every symmetric three-term recurrence equation is a special discrete symplectic system. The assumptions on the coefficients in this paper are weaker and more natural than those in the current literature. In addition, our result implies that symmetric three-term recurrence equations are completely equivalent with Jacobi difference equations arising in the discrete calculus of variations. Presented applications of this study include the Riccati equation and inequality, detailed Sturmian separation and comparison theorems, and the eigenvalue theory for these three-term recurrence and Jacobi equations.

\section{Introduction}

In this paper, we consider the symmetric three-term recurrence equation

$$
S_{k+1} x_{k+2}-T_{k+1} x_{k+1}+S_{k}^{T} x_{k}=0, \quad k \in[0, N-1]_{\mathbb{Z}}
$$

where $x_{k} \in \mathbb{R}^{n}$ for $k \in[0, N+1]_{\mathbb{Z}}$, the real $n \times n$ matrices $S_{k}$ and $T_{k}$ are defined on $[0, N]_{\mathbb{Z}}$ with $T_{k}$ being symmetric and $S_{k}$ being invertible. The discrete intervals are defined by $[a, b]_{\mathbb{Z}}:=[a, b] \cap \mathbb{Z}$. Traditionally, the recurrence equation $(\mathrm{T})$ is studied in the literature; see, for example, [1, Chapter 5] or [2-4], as a generalization of the Jacobi difference equation

$$
\Delta\left(R_{k} \Delta x_{k}+Q_{k}^{T} x_{k+1}\right)=P_{k} x_{k+1}+Q_{k} \Delta x_{k}, \quad k \in[0, N-1]_{\mathbb{Z}}
$$


where $\Delta x_{k}:=x_{k+1}-x_{k}$ is the forward difference and where the matrices $P_{k}, Q_{k}, R_{k} \in \mathbb{R}^{n \times n}$ for $k \in[0, N]_{\mathbb{Z}}$ with $P_{k}$ and $R_{k}$ being symmetric. Jacobi equation (J) arises in the discrete calculus of variations as the Euler equation for the second variation; see, for example, [1, Section 4.2] or [5]. When the forward differences in $(\mathrm{J})$ are expanded, then $(\mathrm{J})$ becomes the three-term recurrence equation $(\mathrm{T})$ in which the matrices $S_{k}:=R_{k}+Q_{k}^{T}$ are invertible for all $k \in[0, N]_{\mathbb{Z}}$ and $T_{k}:=R_{k}+R_{k-1}+Q_{k-1}+Q_{k-1}^{T}+P_{k-1}$ are symmetric; see [1, Section 3.6] or Proposition 2.4. In the same reference, it is shown that Jacobi difference equations $(\mathrm{J})$ and recurrence equations (T) can be embedded into discrete symplectic systems (see Section 2 for the details)

$$
x_{k+1}=\mathbb{A}_{k} x_{k}+\mathbb{B}_{k} u_{k}, \quad u_{k+1}=\mathbb{C}_{k} x_{k}+\mathbb{D}_{k} u_{k}, \quad k \in[0, N]_{\mathbb{Z}}
$$

where for $k \in[0, N]_{\mathbb{Z}}$

$$
\mathbb{S}_{k}^{T} \partial \mathbb{S}_{k}=\partial, \quad \mathbb{S}_{k}:=\left(\begin{array}{ll}
\mathbb{A}_{k} & \mathbb{B}_{k} \\
\mathbb{C}_{k} & \mathbb{D}_{k}
\end{array}\right), \quad \partial:=\left(\begin{array}{cc}
0 & I \\
-I & 0
\end{array}\right)
$$

that is, the $2 n \times 2 n$ matrices $\mathbb{S}_{k}$ are symplectic. However, the transition from $(\mathrm{J})$ and $(\mathrm{T})$ into $(\mathbb{S})$ in reference [1] requires that both $S_{k}$ and $R_{k}$ be invertible. This invertibility assumption essentially means that these equations are first transformed into a linear Hamiltonian system

$$
\Delta x_{k}=A_{k} x_{k+1}+B_{k} u_{k}, \quad \Delta u_{k}=C_{k} x_{k+1}-A_{k}^{T} u_{k}, \quad k \in[0, N]_{\mathbb{Z}}
$$

for which it is required that $I-A_{k}=R_{k}^{-1} S_{k}$ be invertible so that the solutions of (H) exist in the backward time. And then the linear Hamiltonian system $(\mathrm{H})$ is written as the symplectic system $(\mathbb{S})$.

Recently in [6], the authors proposed to study the Jacobi equations (J) as discrete symplectic systems $(\mathbb{S})$ in a direct way which bypasses the Hamiltonian system $(\mathrm{H})$. This new approach requires that only the matrices $S_{k}$ be invertible while the matrices $R_{k}$ are allowed to be singular, which yields more general results for $(\mathrm{J})$ obtained, for example, through the theory of symplectic systems $(\mathbb{S})$. In the present paper, we continue in this direction and we show that the three-term recurrence equations $(\mathrm{T})$ naturally possess a symplectic structure (Theorem 3.1 and Corollary 3.2). More precisely, we show that symmetric threeterm recurrences $(\mathrm{T})$ and Jacobi equations $(\mathrm{J})$ and symplectic systems $(\mathbb{S})$ (with $\mathbb{B}_{k}$ invertible) are completely equivalent. Therefore, the general theory of discrete symplectic systems recently developed, for example, in [7-18] can be applied to obtain, in particular, the Riccati equations and inequalities, and the oscillation and Sturmian theorems (including multiplicities of focal points) for the symmetric three-term recurrence equations (T).

The paper is divided as follows. In the next section, we present an overview of the known transformations between the equations $(\mathrm{T})$, $(\mathrm{J})$, and system $(\mathbb{S})$. In Section 3 we prove the main results about the symplectic structure of the recurrence equation $(\mathrm{T})$. In Sections $4-6$, we present recent results from the theory of discrete symplectic systems $(\mathbb{S})$ adopted for the setting of recurrence equations $(\mathrm{T})$. These results include the Riccati equations and inequalities as being a part of the Reid roundabout theorems in Section 4, the Sturmian separation and comparison theorems in Section 5, and the oscillation theorems and Rayleigh principle in Section 6. In Section 7, we make some final comments about the results of this paper. 


\section{Known Results}

We first present known transformations between the three-term recurrence equation $(T)$, Jacobi equation $(\mathrm{J})$, and the symplectic system $(\mathbb{S})$. We adopt the following notation.

Notation 2.1 (Three-term recurrence (T)). The matrices $S_{k}, T_{k}$, and the vectors $x_{k}$ in $(\mathrm{T})$ have the following properties: $S_{k}, T_{k} \in \mathbb{R}^{n \times n}$ are defined on $[0, N+1]_{\mathbb{Z}}$ with $S_{k}$ invertible and $T_{k}$ symmetric; $x_{k} \in \mathbb{R}^{n}$ are defined on $[0, N+1]_{\mathbb{Z}}$.

Notation 2.2 (Jacobi equation (J)). The matrices $P_{k}, Q_{k}, R_{k}$, and the vectors $x_{k}$ in (J) have the following properties: $P_{k}, Q_{k}, R_{k} \in \mathbb{R}^{n \times n}$ are defined on $[0, N]_{\mathbb{Z}}, P_{k}$ and $R_{k}$ are symmetric, and the matrix $S_{k}:=R_{k}+Q_{k}^{T}$ is invertible; $x_{k} \in \mathbb{R}^{n}$ for $k$ in $[0, N+1]_{\mathbb{Z}}$.

Note that the coefficients $T_{0}, S_{N+1}$, and $T_{N+1}$ are not explicitly needed in $(\mathrm{T})$ and the coefficient $P_{N}$ is not needed in (J). However, it will be convenient to use them when we transform $(\mathrm{T})$ into $(\mathrm{J})$ or system $(\mathbb{S})$ and vice versa. For example, we can now define $x_{N+2}:=S_{N+1}^{-1}\left(T_{N+1} x_{N+1}-S_{N}^{T} x_{N}\right)$, so that the recurrence in (T) is satisfied also at $t=N$.

Notation 2.3 (Symplectic system (S)). The matrices $\mathbb{A}_{k}, \mathbb{B}_{k}, \mathbb{C}_{k}, \mathbb{D}_{k}$, and the vectors $x_{k}, u_{k}$ in $(\mathbb{S})$ have the following properties: $\mathbb{A}_{k}, \mathbb{B}_{k}, \mathbb{C}_{k}, \mathbb{D}_{k} \in \mathbb{R}^{n \times n}$ are defined on $[0, N]_{\mathbb{Z}}$ and satisfy (1.1); $x_{k}, u_{k} \in \mathbb{R}^{n}$ are defined for $k$ in $[0, N+1]_{\mathbb{Z}}$.

The following two known results are verified by straightforward calculations. They can be found in [1, Section 3.6].

Proposition 2.4 (Jacobi (J) to three-term recurrence (T)). Assume that $P_{k}, Q_{k}, R_{k}, S_{k}:=R_{k}+Q_{k}^{T}$ satisfy the conditions in Notation 2.2 and set $R_{N+1}:=I$. Then the Jacobi equation $(\mathrm{J})$ is the symmetric three-term recurrence equation $(\mathrm{T})$, whose coefficients

$$
S_{k}:=R_{k}+Q_{k}^{T}, \quad T_{k+1}:=R_{k+1}+R_{k}+Q_{k}+Q_{k}^{T}+P_{k}, \quad k \in[0, N]_{\mathbb{Z}}
$$

satisfy the conditions in Notation 2.1.

Note that the choice of $R_{N+1}:=I$ in Proposition 2.4 is arbitrary, that is, any matrix $R_{N+1} \in \mathbb{R}^{n \times n}$ will do the job.

Proposition 2.5 (Three-term recurrence (T) to Jacobi (J), $R_{k}$ invertible). Assume that $S_{k}$, $T_{k}$ satisfy the conditions in Notation 2.1. Let $R_{k}$ be any symmetric and invertible matrices for $k \in[0, N+1]_{\mathbb{Z}}$. Then the symmetric three-term recurrence equation $(\mathrm{T})$ is the Jacobi equation $(\mathrm{J})$, whose coefficients

$$
R_{k}, Q_{k}:=S_{k}^{T}-R_{k}, \quad P_{k}:=T_{k+1}-S_{k}^{T}-S_{k}+R_{k}-R_{k+1}, \quad k \in[0, N]_{\mathbb{Z}}
$$

satisfy the conditions in Notation 2.2. 
Remark 2.6. When the matrix $S_{k}$ is also symmetric, we may take $R_{k}:=S_{k}$ in Proposition 2.5. It follows that the recurrence equation (T) is then transformed into the Jacobi equation $(\mathrm{J})$, whose coefficients

$$
R_{k}:=S_{k}, \quad Q_{k}:=0, \quad P_{k}:=T_{k+1}-S_{k+1}-S_{k}, \quad k \in[0, N]_{\mathbb{Z}}
$$

satisfy the conditions in Notation 2.2. Note that in this case the matrix $R_{k}=S_{k}$ is invertible.

The invertibility condition on $R_{k}$ in Proposition 2.5 or Remark 2.6 means that the resulting Jacobi equation can be written as a linear Hamiltonian system $(\mathrm{H})$, which in turn can be written as a symplectic system $(\mathbb{S})$. This is shown in [1, Example 3.17].

Proposition 2.7 (Jacobi (J) to symplectic $(\mathbb{S}), R_{k}$ invertible). Assume that $P_{k}, Q_{k}, R_{k}, S_{k}:=$ $R_{k}+Q_{k}^{T}$ satisfy the conditions in Notation 2.2 with $R_{k}$ being invertible. Then the Jacobi equation (J) is the symplectic system $(\mathbb{S})$, whose coefficients

$$
\begin{gathered}
\mathbb{A}_{k}:=S_{k}^{-1} R_{k}, \quad \mathbb{C}_{k}:=\left(P_{k}-Q_{k} R_{k}^{-1} Q_{k}^{T}\right) S_{k}^{-1} R_{k}, \quad k \in[0, N]_{\mathbb{Z}} \\
\mathbb{B}_{k}:=S_{k}^{-1}, \quad \mathbb{D}_{k}:=\left(P_{k}-Q_{k} R_{k}^{-1} Q_{k}^{T}\right) S_{k}^{-1}+I+Q_{k} R_{k}^{-1}, \quad k \in[0, N]_{\mathbb{Z}}
\end{gathered}
$$

with $u_{k}:=S_{k} x_{k+1}-R_{k} x_{k}$ on $[0, N]_{\mathbb{Z}}$ and $u_{N+1}:=\left(P_{N}+Q_{N}+S_{N}\right) x_{N+1}-S_{N}^{T} x_{N}$ satisfy the conditions in Notation 2.3.

It is interesting to observe that by using the identity $Q_{k}^{T}=S_{k}-R_{k}$ one can eliminate the inverse of $R_{k}$ in the coefficients (2.4) to obtain the coefficients in Proposition 2.8 below. This was actually the motivation for the investigation of Jacobi systems as discrete symplectic systems in [6]. In this latter reference, the authors showed that it is possible to treat Jacobi equation $(\mathrm{J})$ directly as a symplectic system $(\mathbb{S})$ by bypassing the Hamiltonian system $(\mathrm{H})$; see [6, Corollary 5.2].

Proposition 2.8 (Jacobi (J) to symplectic $(\mathbb{S})$ ). Assume that $P_{k}, Q_{k}, R_{k}, S_{k}:=R_{k}+Q_{k}^{T}$ satisfy the conditions in Notation 2.2. Then the Jacobi equation $(\mathrm{J})$ is the symplectic system $(\mathbb{S})$, whose coefficients

$$
\begin{aligned}
\mathbb{A}_{k}:=S_{k}^{-1} R_{k}, \quad \mathbb{C}_{k}:=P_{k} S_{k}^{-1} R_{k}-Q_{k} S_{k}^{-1} Q_{k}^{T}, & k \in[0, N]_{\mathbb{Z}} \\
\mathbb{B}_{k}:=S_{k}^{-1}, \quad \mathbb{D}_{k}:=\left(P_{k}+Q_{k}+Q_{k}^{T}+R_{k}\right) S_{k}^{-1}, & k \in[0, N]_{\mathbb{Z}}
\end{aligned}
$$

with $u_{k}:=S_{k} x_{k+1}-R_{k} x_{k}$ on $[0, N]_{\mathbb{Z}}$ and $u_{N+1}:=\left(P_{N}+Q_{N}+S_{N}\right) x_{N+1}-S_{N}^{T} x_{N}$ satisfy the conditions in Notation 2.3. Moreover, the resulting symplectic system $(\mathbb{S})$ is Hamiltonian if and only if the matrix $R_{k}$ is invertible.

The resulting symplectic system in Proposition 2.8 has $\mathbb{B}_{k}=S_{k}^{-1}$ invertible. This turns out to be a characterizing property of symplectic systems $(\mathbb{S})$ corresponding to Jacobi equations (J); see [6, Corollary 5.3]. 
Proposition 2.9 (Symplectic ( $\mathbb{S}$ ) to Jacobi (J)). Assume that $\mathbb{A}_{k}, \mathbb{B}_{k}, \mathbb{C}_{k}, \mathbb{D}_{k}$ satisfy the conditions in Notation 2.3 with $\mathbb{B}_{k}$ being invertible. Then the symplectic system $(\mathbb{S})$ is the Jacobi equation $(\mathrm{J})$, whose coefficients

$$
R_{k}:=\mathbb{B}_{k}^{-1} \mathbb{A}_{k}, \quad Q_{k}:=\left(I-\mathbb{A}_{k}^{T}\right) \mathbb{B}_{k}^{T-1}, \quad P_{k}:=\left(\mathbb{D}_{k}-I\right) \mathbb{B}_{k}^{-1}+\left(\mathbb{A}_{k}^{T}-I\right) \mathbb{B}_{k}^{T-1}, \quad k \in[0, N]_{\mathbb{Z}}
$$

with $S_{k}=R_{k}+Q_{k}^{T}=\mathbb{B}_{k}^{-1}$ satisfy the conditions in Notation 2.2.

Next we turn our attention back to the symmetric three-term recurrence equations $(\mathrm{T})$. By combining the transformations in Propositions 2.5 and 2.8 we get the following.

Proposition 2.10 (Three-term recurrence $(\mathrm{T})$ to symplectic $(\mathbb{S}), R_{k}$ invertible). Assume that $S_{k}, T_{k}$ satisfy the conditions in Notation 2.1. Let $R_{k}$ be any symmetric and invertible matrices for $k \in[0, N+1]_{\mathbb{Z}}$. Then the symmetric three-term recurrence equation $(\mathrm{T})$ is the symplectic system $(\mathbb{S})$, whose coefficients

$$
\begin{gathered}
\mathbb{A}_{k}:=S_{k}^{-1} R_{k}, \quad \mathbb{C}_{k}:=\left(T_{k+1}-R_{k+1}\right) S_{k}^{-1} R_{k}-S_{k}^{T}, \quad k \in[0, N]_{\mathbb{Z}} \\
\mathbb{B}_{k}:=S_{k}^{-1}, \quad \mathbb{D}_{k}:=\left(T_{k+1}-R_{k+1}\right) S_{k}^{-1}, \quad k \in[0, N]_{\mathbb{Z}}
\end{gathered}
$$

with $u_{k}:=S_{k} x_{k+1}-R_{k} x_{k}$ on $[0, N]_{\mathbb{Z}}$ and $u_{N+1}:=\left(T_{N+1}-R_{N+1}\right) x_{N+1}-S_{N}^{T} x_{N}$ satisfy the conditions in Notation 2.3.

\section{Main Results}

The need to have $R_{k}$ invertible in Proposition 2.10 is artificial, because $R_{k}$ is not furnished by the three-term recurrence equation (T) and furthermore, $R_{k}^{-1}$ is not even present in equations (2.7) which define the coefficients of the corresponding symplectic system $(\mathbb{S})$. However, the invertibility of $R_{k}$ is a requirement inherited from Proposition 2.5 that was derived in [1, Section 3.6]. Therefore, an important question naturally surfaces: is it possible to obtain the result of Propositions 2.5 and 2.10 without any assumption on $R_{k}$ ?

The following new result provides an answer to the above question, that is, it shows that the recurrence equations $(\mathrm{T})$ are naturally special cases of symplectic systems $(\mathbb{S})$ for any choice of matrices $R_{k}$ and without any assumption on the invertibility of $T_{k}$.

Theorem 3.1 (Three-term recurrence (T) to symplectic ( $\mathbb{S}$ ), $R_{k}$ arbitrary). Assume that $S_{k}, T_{k}$ satisfy the conditions in Notation 2.1. Let $R_{k}$ be any symmetric matrices for $k \in[0, N+1]_{\mathbb{Z}}$. Then the symmetric three-term recurrence equation $(\mathrm{T})$ is the symplectic system $(\mathbb{S})$, whose coefficients are given by (2.7) with $u_{k}:=S_{k} x_{k+1}-R_{k} x_{k}$ on $[0, N]_{\mathbb{Z}}$ and $u_{N+1}:=\left(T_{N+1}-R_{N+1}\right) x_{N+1}-S_{N}^{T} x_{N}$ and they satisfy the conditions in Notation 2.3.

Proof. Given (T) with the data as in Notation 2.1, we set $u_{k}:=S_{k} x_{k+1}-R_{k} x_{k}$ for $k \in[0, N]_{\mathbb{Z}}$ and $u_{N+1}:=\left(T_{N+1}-R_{N+1}\right) x_{N+1}-S_{N}^{T} x_{N}$. Then

$$
\begin{aligned}
x_{k+1} & =S_{k}^{-1} R_{k} x_{k}+S_{k}^{-1} u_{k}=\mathbb{A}_{k} x_{k}+\mathbb{B}_{k} u_{k}, \quad k \in[0, N]_{\mathbb{Z}} \\
u_{k+1} & =S_{k+1} x_{k+2}-R_{k+1} x_{k+1} \stackrel{(T)}{=}\left(T_{k+1}-R_{k+1}\right) x_{k+1}-S_{k}^{T} x_{k} \\
& =\mathbb{C}_{k} x_{k}+\mathbb{D}_{k} u_{k}, \quad k \in[0, N-1]_{\mathbb{Z}} .
\end{aligned}
$$


Therefore, with the coefficients $\mathbb{A}_{k}, \mathbb{B}_{k}, \mathbb{C}_{k}$, and $\mathbb{D}_{k}$ defined by (2.7), we have that the pair $(x, u)$ satisfies the first equation in system $(\mathbb{S})$ for all $k \in[0, N]_{\mathbb{Z}}$ and the second equation in (S) for all $k \in[0, N-1]_{\mathbb{Z}}$. However, the definition of $u_{N+1}$ yields that the second equation in $(\mathbb{S})$ holds also at $k=N$. It remains to show that the matrix $\mathbb{S}_{k}$, defined in (1.1) through $\mathbb{A}_{k}, \mathbb{B}_{k}$, $\mathbb{C}_{k}$, and $\mathbb{D}_{k}$ in (2.7), is symplectic. We have after easy calculations that for every $k \in[0, N]_{\mathbb{Z}}$,

$$
\mathbb{S}_{k}^{T} \partial \mathbb{S}_{k}=\left(\begin{array}{ll}
\mathbb{A}_{k} & \mathbb{B}_{k} \\
\mathbb{C}_{k} & \mathbb{D}_{k}
\end{array}\right)^{T}\left(\begin{array}{cc}
0 & I \\
-I & 0
\end{array}\right)\left(\begin{array}{ll}
\mathbb{A}_{k} & \mathbb{B}_{k} \\
\mathbb{C}_{k} & \mathbb{D}_{k}
\end{array}\right)=\partial,
$$

where we used the symmetry of $R_{k}$. The proof is complete.

Note that the matrices $R_{k}$ in the above theorem are arbitrary, and hence, one can choose them to simplify the formulas of the coefficients in (2.7). One choice standing out is when $R_{k}=T_{k}$. In this case, the result of Theorem 3.1 reduces to the following.

Corollary 3.2 (Three-term recurrence $(\mathrm{T})$ to symplectic $(\mathbb{S})$ ). Assume that $S_{k}, T_{k}$ satisfy the conditions in Notation 2.1. Then the symmetric three-term recurrence equation $(\mathrm{T})$ is the symplectic system $(\mathbb{S})$, whose coefficients

$$
\mathbb{A}_{k}:=S_{k}^{-1} T_{k}, \quad \mathbb{B}_{k}:=S_{k}^{-1}, \quad \mathbb{C}_{k}:=-S_{k}^{T}, \quad \mathbb{D}_{k}:=0, \quad k \in[0, N]_{\mathbb{Z}},
$$

with $u_{k}:=S_{k} x_{k+1}-T_{k} x_{k}$ on $[0, N]_{\mathbb{Z}}$ and $u_{N+1}:=-S_{N}^{T} x_{N}$ satisfy the conditions in Notation 2.3.

The above result has an important consequence. By using Proposition 2.9, we can now transform any symmetric three-term recurrence equation $(\mathrm{T})$ into a Jacobi equation (J) by the procedure described in Corollary 3.2 and Proposition 2.9. However, compared with the result in Proposition 2.5, we do not need the matrices $R_{k}$ to be invertible, but they can be arbitrary.

Corollary 3.3 (Three-term recurrence (T) to Jacobi (J)). Assume that $S_{k}, T_{k}$ satisfy the conditions in Notation 2.1. Let $R_{k}$ be any symmetric matrices for $k \in[0, N+1]_{\mathbb{Z}}$. Then the symmetric three-term recurrence equation $(\mathrm{T})$ is the Jacobi equation $(\mathrm{J})$, whose coefficients are given by equations (2.2) and they satisfy the conditions in Notation 2.2. When $R_{k}:=T_{k}$, the coefficients of $(\mathrm{J})$ reduce to

$$
R_{k}:=T_{k}, \quad Q_{k}:=S_{k}^{T}-T_{k}, \quad P_{k}:=T_{k}-S_{k}-S_{k}^{T}, \quad k \in[0, N]_{\mathbb{Z}} .
$$

Note that if the matrices $R_{k}$ are invertible, then Corollary 3.3 is a consequence of Proposition 2.5. This is also the case for the second part of the Corollary 3.3 if $T_{k}$ are invertible. result.

When combining the transformations in Propositions 2.9 and 2.4, we get the following

Corollary 3.4 (Symplectic (S) to three-term recurrence (T)). Assume that $\mathbb{A}_{k}, \mathbb{B}_{k}, \mathbb{C}_{k}, \mathbb{D}_{k}$ satisfy the conditions in Notation 2.3 with $\mathbb{B}_{k}$ being invertible and set $\mathbb{A}_{N+1}=\mathbb{B}_{N+1}:=I$. Then the symplectic system $(\mathbb{S})$ is the the symmetric three-term recurrence equation $(\mathrm{T})$, whose coefficients

$$
S_{k}:=\mathbb{B}_{k}^{-1}, \quad T_{k+1}:=\mathbb{B}_{k+1}^{-1} \mathbb{A}_{k+1}^{-1}+\mathbb{D}_{k} \mathbb{B}_{k}^{-1}, \quad k \in[0, N]_{\mathbb{Z}},
$$

with $T_{0}=\mathbb{B}_{0}^{-1} \mathbb{A}_{0}$ satisfy the conditions in Notation 2.1. 
In the last part of this section, we pay attention to the quadratic functionals associated with the equations $(\mathrm{T}),(\mathrm{J})$, and system $(\mathbb{S})$. In particular, we show that these functionals transform exactly in the same way as their corresponding systems. Consider the quadratic functionals

$$
\begin{aligned}
& \mathcal{F}_{T}(x):=\sum_{k=0}^{N}\left\{x_{k+1}^{T} T_{k+1} x_{k+1}-x_{k}^{T} S_{k} x_{k+1}-x_{k+1}^{T} S_{k}^{T} x_{k}\right\}, \\
& \mathcal{F}_{J}(x):=\sum_{k=0}^{N}\left\{x_{k+1}^{T} P_{k} x_{k+1}+2 x_{k+1}^{T} Q_{k} x_{k}+\Delta x_{k}^{T} R_{k} \Delta x_{k}\right\}, \\
& \mathbb{F}(x, u):=\sum_{k=0}^{N}\left\{x_{k}^{T} \mathbb{C}_{k}^{T} \mathbb{A}_{k} x_{k}+2 x_{k}^{T} \mathbb{C}_{k}^{T} \mathbb{B}_{k} u_{k}+u_{k}^{T} \mathbb{D}_{k}^{T} \mathbb{B}_{k} u_{k}\right\},
\end{aligned}
$$

where $x=\left\{x_{k}\right\}_{k=0}^{N+1}$ satisfies $x_{0}=0=x_{N+1}$. In addition, in functional $\mathbb{F}$ the pair $(x, u)$ solves the first equation in system $(\mathbb{S})$, that is, $x_{k+1}=\mathbb{A}_{k} x_{k}+\mathbb{B}_{k} u_{k}$ for $k \in[0, N]_{\mathbb{Z}}$. The following result is from [6, Proposition 3.7].

Proposition 3.5 (Quadratic functionals for $(\mathrm{J})$ and $(\mathbb{S})$ ). Assume that

(i) either $P_{k}, Q_{k}$, and $R_{k}$ satisfy the conditions in Notation 2.2 with $\mathbb{A}_{k}, \mathbb{B}_{k}, \mathbb{C}_{k}, \mathbb{D}_{k}$, and $u_{k}$ being given by (2.5) of Proposition 2.8

(ii) or $\mathbb{A}_{k}, \mathbb{B}_{k}, \mathbb{C}_{k}$, and $\mathbb{D}_{k}$ satisfy the conditions in Notation 2.3 with $\mathbb{B}_{k}$ being invertible and $P_{k}, Q_{k}, R_{k}$ are given by (2.6).

Then $\mathcal{F}_{J}(x)=\mathbb{F}(x, u)$ for every $x=\left\{x_{k}\right\}_{k=0}^{N+1}$ with $x_{0}=0=x_{N+1}$.

As a consequence of the results in Theorem 3.1 and Corollary 3.4, and in Proposition 2.4 and Corollary 3.3, we get the transformations between the functionals $\mathcal{F}_{T}$ and $\mathbb{F}$, and $\mathcal{F}_{T}$ and $\mathcal{F}_{J}$.

Proposition 3.6 (Quadratic functionals for $(\mathrm{T})$ and $(\mathbb{S})$ ). Assume that

(i) either $S_{k}, T_{k}$ satisfy the conditions in Notation 2.1 with $\mathbb{A}_{k}, \mathbb{B}_{k}, \mathbb{C}_{k}, \mathbb{D}_{k}$, and $u_{k}$ being given by (2.7) of Theorem 3.1

(ii) or $\mathbb{A}_{k}, \mathbb{B}_{k}, \mathbb{C}_{k}$, and $\mathbb{D}_{k}$ satisfy the conditions in Notation 2.3 with $\mathbb{B}_{k}$ being invertible and $T_{k}, S_{k}$ are given by (3.5).

Then $\mathcal{F}_{T}(x)=\mathbb{F}(x, u)$ for every $x=\left\{x_{k}\right\}_{k=0}^{N+1}$ with $x_{0}=0=x_{N+1}$.

Proposition 3.7 (Quadratic functionals for $(\mathrm{J})$ and $(\mathrm{T})$ ). Assume that

(i) either $P_{k}, Q_{k}$, and $R_{k}$ satisfy the conditions in Notation 2.2 with $T_{k}, S_{k}$ being given by (2.1)

(ii) or $T_{k}, S_{k}$ satisfy the conditions in Notation 2.1 with $S_{k}$ being invertible and $P_{k}, Q_{k}, R_{k}$ are given by (3.4).

Then $\mathcal{F}_{T}(x)=\mathcal{F}_{J}(x)$ for every $x=\left\{x_{k}\right\}_{k=0}^{N+1}$ with $x_{0}=0=x_{N+1}$. 


\section{Applications in Reid Roundabout Theorems}

In the previous section, we proved that symmetric three-term recurrence equations $(\mathrm{T})$ and Jacobi difference equations $(\mathrm{J})$ are completely equivalent, that is, any result for one equation (T) or $(\mathrm{J})$ can be translated via the transformations in Proposition 2.4 and Corollary 3.3 to a result for the other equation. This equivalence is carried over via discrete symplectic systems $\left(\mathbb{S}\right.$ ) with $\mathbb{B}_{k}$ being invertible, utilizing the transformations in Propositions 2.8 and 2.9 (for the passage between equation $(\mathrm{J})$ and system $(\mathbb{S})$ ) and the transformations in Theorem 3.1 and Corollary 3.4 (for the passage between equation $(\mathrm{T})$ and system $(\mathbb{S})$ ). In this section, we give some applications of this equivalence. For example, we derive the Riccati equation and Riccati inequality which are naturally associated with the symmetric three-term recurrence equations $(\mathrm{T})$ - the results which have not been known in the literature for $(\mathrm{T})$.

Consider the quadratic functional $\mathcal{F}_{T}(x)$ defined in (3.6) subject to sequences $x=$ $\left\{x_{k}\right\}_{k=0}^{N+1}$ satisfying $x_{0}=0=x_{N+1}$. Note that due to $x_{N+1}=0$, the functional $\mathcal{F}_{T}$ does not depend on the matrix $T_{N+1}$, as we mentioned at the beginning of Section 2 . We say that the functional $\mathcal{F}_{T}$ is positive definite if $\mathcal{F}_{T}(x)>0$ for every $x=\left\{x_{k}\right\}_{k=0}^{N+1}$ with $x_{0}=0=x_{N+1}$ and $x \neq 0$. We say that $\mathcal{F}_{T}$ is nonnegative if $\mathcal{F}_{T}(x) \geq 0$ for every $x=\left\{x_{k}\right\}_{k=0}^{N+1}$ with $x_{0}=0=x_{N+1}$.

The positivity of the functional $\mathscr{f}_{T}$ was first characterized in [2, Theorem 4]; see also [1, Theorem 5.13] and [4, Corollary 2], in terms of the properties of the so-called conjoined bases of $(\mathrm{T})$. These are the $n \times n$ matrix solutions $X=\left\{X_{k}\right\}_{k=0}^{N+1}$ of $(\mathrm{T})$ such that $X_{k}^{T} S_{k} X_{k+1}$ is symmetric and $\operatorname{rank}\left(X_{k}^{T} \quad X_{k+1}^{T}\right)=n$ for some (and hence for any) index $k \in[0, N]_{\mathbb{Z}}$. A special conjoined basis $\widehat{X}$ of $(\mathrm{T})$, determined by the initial conditions $\widehat{X}_{0}=0$ and $\widehat{X}_{1}=S_{0}^{-1}$, is called the principal solution of $(\mathrm{T})$.

Proposition 4.1 (Reid roundabout theorem-positivity). Assume that $S_{k}, T_{k}$ satisfy the conditions in Notation 2.1. Then the following statements are equivalent.

(i) The functional $\boldsymbol{F}_{T}$ is positive definite.

(ii) The principal solution $\widehat{X}$ of $(\mathrm{T})$ has $\widehat{X}_{k}$ invertible for all $k \in[1, N+1]_{\mathbb{Z}}$ and satisfies $\widehat{X}_{k}^{T} S_{k} \widehat{X}_{k+1}>0$ for all $k \in[1, N]_{\mathbb{Z}}$.

(iii) There exists a conjoined basis $X$ of $(T)$ such that $X_{k}$ is invertible for all $k \in[0, N+1]_{\mathbb{Z}}$ and satisfying $X_{k}^{T} S_{k} X_{k+1}>0$ for all $k \in[0, N]_{\mathbb{Z}}$.

Proof. See [1, Theorem 5.13] or [4, Corollary 2].

A similar result holds for the nonnegativity of $\mathcal{F}_{T}$.

Proposition 4.2 (Reid roundabout theorem-nonnegativity). Assume that $S_{k}, T_{k}$ satisfy the conditions in Notation 2.1. Then the following statements are equivalent.

(i) The functional $\mathscr{f}_{T}$ is nonnegative.

(ii) The principal solution $\widehat{X}$ of $(\mathrm{T})$ has $\widehat{X}_{k}$ invertible for all $k \in[1, N]_{\mathbb{Z}}$ and satisfies $\widehat{X}_{k}^{T} S_{k} \widehat{X}_{k+1}>0$ for all $k \in[1, N-1]_{\mathbb{Z}}$ and $\widehat{X}_{N}^{T} S_{N} \widehat{X}_{N+1} \geq 0$.

Proof. See [4, Theorem 2]. 
In the following result, we add three more equivalent conditions to Proposition 4.1 and one more equivalent condition to Proposition 4.2 in terms of solutions of the discrete Riccati matrix equation and inequality corresponding to $(\mathrm{T})$, thus completing the above results to their full standard forms. For symmetric matrices $W=\left\{W_{k}\right\}_{k=0}^{N+1}$, define the Riccati operator

$$
R[W]_{k}:=W_{k+1} S_{k}^{-1}\left(T_{k}+W_{k}\right)+S_{k}^{T}, \quad k \in[0, N]_{\mathbb{Z}}
$$

This Riccati operator is obtained from the Riccati operator $\mathbb{R}[W]_{k}:=W_{k+1}\left(\mathbb{A}_{k}+\mathbb{B}_{k} W_{k}\right)-\left(\mathbb{C}_{k}+\right.$ $\mathbb{D}_{k} W_{k}$ ) for symplectic system $(\mathbb{S})$; see, for example, $[7,14-16,19]$. That is, if the coefficients of $(\mathbb{S})$ are given by formulas (3.3), then $\mathbb{R}[W]_{k}=R[W]_{k}$. The equation $R[W]_{k}=0$ for $k \in$ $[0, N]_{\mathbb{Z}}$ is called the discrete Riccati matrix equation. If $T_{k}+W_{k}$ is invertible, then we may solve the equation $R[W]_{k}=0$ for $W_{k+1}$ and obtain the (symmetric) Riccati equation corresponding to the recurrence equation $(\mathrm{T})$, that is,

$$
W_{k+1}+S_{k}^{T}\left(T_{k}+W_{k}\right)^{-1} S_{k}=0, \quad k \in[0, N]_{\mathbb{Z}}
$$

The Riccati equation (RE) has been studied in the literature by many authors; see, for example, the references discussed in [20, page 12]. However, its natural connection to the recurrence equation $(\mathrm{T})$ is established for the first time in this paper. In addition, the discrete Riccati inequality $\mathbb{R}[W]_{k}\left(\mathbb{A}_{k}+\mathbb{B}_{k} W_{k}\right)^{-1} \leq 0$ derived in [14, Theorem 1$]$ for symplectic systems $(\mathbb{S})$ yields through Corollary 3.2 a new Riccati inequality

$$
W_{k+1}+S_{k}^{T}\left(T_{k}+W_{k}\right)^{-1} S_{k} \leq 0, \quad k \in[0, N]_{\mathbb{Z}}
$$

for the recurrence equation (T). Equivalently, the Riccati equation (RE) and inequality (RI) can be obtained from the Riccati equation and inequality for the Jacobi equation

$$
\Delta W_{k}-P_{k}+\left(W_{k}-Q_{k}\right)\left(R_{k}+W_{k}\right)^{-1}\left(W_{k}-Q_{k}^{T}\right)=0,(\leq 0) \quad k \in[0, N]_{\mathbb{Z}}
$$

in which the coefficients are given by the formulas in (3.4). Note that discrete Riccati equations obtained from symplectic system $(\mathbb{S})$ corresponding to three-term recurrence equations (T) with $R_{k}$ being invertible (as in Propositions 2.5 and 2.10) are considered in [20], [1, Section 6.1], and [16, Section 4]. In Theorem 4.3 below, we do not require any condition on $R_{k}$. The following result is a complement of Proposition 4.1.

Theorem 4.3 (Reid roundabout theorem-positivity (continued)). Assume that $S_{k}, T_{k}$ satisfy the conditions in Notation 2.1. Then each of the conditions (i)-(iii) of Proposition 4.1 is equivalent to any of the following statements.

(iv) There exists a symmetric solution $W_{k}$ on $[0, N+1]_{\mathbb{Z}}$ of the Riccati equation (RE) for $k \in[1, N]_{\mathbb{Z}}$ such that $W_{0}=0$ and $T_{k}+W_{k}>0$ for all $k \in[1, N]_{\mathbb{Z}}$.

(v) There exists a symmetric solution $W_{k}$ on $[0, N+1]_{\mathbb{Z}}$ of the Riccati equation (RE) such that $T_{k}+W_{k}>0$ for all $k \in[0, N]_{\mathbb{T}}$.

(vi) There exists a symmetric solution $W_{k}$ on $[0, N+1]_{\mathbb{Z}}$ of the Riccati inequality (RI) satisfying $T_{k}+W_{k}>0$ for all $k \in[0, N]_{\mathbb{T}}$. 
Proof. With the coefficients of system $(\mathbb{S})$ given by (3.3), the equivalence of conditions (i) and (v) is established in [16, Theorem 7] and the equivalence of (i) and (vi) in [14, Theorem 1]. Condition (ii) of Proposition 4.1 implies condition (iv) by setting $W_{0}:=0, W_{k}:=S_{k} \widehat{X}_{k+1} \widehat{X}_{k}^{-1}-$ $T_{k}$ for $k \in[1, N]_{\mathbb{Z}}$, and $W_{N+1}:=-S_{N}^{T} \widehat{X}_{N} \widehat{X}_{N+1}^{-1}$ (i.e., we set $W_{k}:=\widehat{U}_{k} \widehat{X}_{k}^{-1}$ on $[1, N+1]_{\mathbb{Z}}$, where $\widehat{U}_{k}:=S_{k} \widehat{X}_{k+1}-T_{k} \widehat{X}_{k}$ for $k \in[0, N]_{\mathbb{Z}}$ and $\widehat{U}_{N+1}:=-S_{N}^{T} \widehat{X}_{N}$ accordingly to the definition of $u_{k}$ in Theorem 3.1). Then $T_{k}+W_{k}=\widehat{X}_{k}^{T-1}\left(\widehat{X}_{k}^{T} S_{k} \widehat{X}_{k+1}\right) \widehat{X}_{k}^{-1}>0$ for all $k \in[1, N]_{\mathbb{Z}}$. Hence, condition (iv) is satisfied. Finally, if we assume that condition (iv) holds, then the proof of the positivity of $\mathfrak{F}_{T}$ is similar to the proof of [4, Corollary 3].

Note that condition (vi) of Theorem 4.3 yields that $-W_{k+1} \geq S_{k}^{T}\left(T_{k}+W_{k}\right)^{-1} S_{k}>0$, which implies that the matrices $W_{k}$ in Theorem 4.3(vi) are negative definite for $k \in[1, N+1]_{\mathbb{Z}}$.

The second result of this section is concerned with the nonnegativity of the functional $\mathcal{F}_{T}$.

Theorem 4.4 (Reid roundabout theorem-nonnegativity (continued)). Assume that $S_{k}, T_{k}$ satisfy the conditions in Notation 2.1. Then each of the conditions (i)-(ii) of Proposition 4.2 is equivalent to the following statement.

(iii) There exists a symmetric solution $W_{k}$ on $[0, N+1]_{\mathbb{Z}}$ of the Riccati equation (RE) for $k \in[1, N-1]_{\mathbb{Z}}$ such that $W_{0}=0, T_{k}+W_{k}>0$ for all $k \in[1, N-1]_{\mathbb{Z}}$, and $T_{N}+W_{N} \geq 0$.

Proof. The proof is similar to implications (ii) $\Rightarrow$ (iv) $\Rightarrow$ (i) from the proof of Theorem 4.3. The details are here therefore omitted. Alternatively, see the proof of [4, Theorem 3].

By comparing the Riccati equation conditions for the positivity and nonnegativity of $\mathcal{F}_{T}$ in Theorem 4.3(iv) and Theorem 4.4(iii), we can see that the Riccati equation for the positivity of $\mathcal{F}_{T}$ is satisfied on the closed interval (including $k=N$ ), while the Riccati equation for the nonnegativity of $\mathcal{F}_{T}$ is satisfied on the open interval (excluding $k=N$ ). This phenomenon resembles the situation in the continuous time setting in [21] or [22, Section 6.2], that is, for Jacobi differential equations or Hamiltonian systems and their corresponding Riccati differential equations.

\section{Applications in Sturmian Theory}

In [1, Sections 5.3 and 5.6], several Sturmian comparison and separation theorems are presented for the symmetric three-term recurrence equations $(\mathrm{T})$. However, these results do not involve the multiplicities of focal points for conjoined bases of $(\mathrm{T})$. Therefore, our next aim is to extend the Sturmian separation and comparison theorems for symmetric three-term recurrence equations $(\mathrm{T})$ in this direction.

Following [18], we say that a conjoined basis $X$ of $(\mathrm{T})$ has a focal point in the point $k+1$ if $X_{k+1}$ is singular and then $\operatorname{def} X_{k+1}:=\operatorname{dim} \operatorname{Ker} X_{k+1}$ is its multiplicity, while the conjoined basis $X$ has a focal point in the interval $(k, k+1)_{\mathbb{Z}}$ if the matrix $X_{k}^{T} S_{k} X_{k+1}$ is not nonnegative definite and then ind $X_{k}^{T} S_{k} X_{k+1}$ is its multiplicity. Here ind $A$ is defined as the number of negative eigenvalues of the (symmetric) matrix $A$. The number of focal points in the interval $(k, k+1]_{\mathbb{Z}}$ (including multiplicities) is then $m_{k}:=\operatorname{def} X_{k+1}+\operatorname{ind} X_{k}^{T} S_{k} X_{k+1}$. We will always count the focal points of conjoined bases of $(\mathrm{T})$ including their multiplicities. This definition of multiplicities is motivated by the appearance of the symmetric matrix $X_{k}^{T} S_{k} X_{k+1}$ in the Reid roundabout theorem (Propositions 4.1 and 4.2). Therefore, the conditions (ii) in Propositions 4.1 and 4.2 can be reformulated as follows. 
Corollary 5.1 (Reid roundabout theorem-positivity (continued)). Assume that $S_{k}, T_{k}$ satisfy the conditions in Notation 2.1. Condition (ii) of Proposition 4.1 has the following equivalent form.

(ii)' The principal solution of $(\mathrm{T})$ has no focal points in the interval $(0, N+1]_{\mathbb{Z}}$.

Corollary 5.2 (Reid roundabout theorem-nonnegativity (continued)). Assume that $S_{k}, T_{k}$ satisfy the conditions in Notation 2.1. Condition (ii) of Proposition 4.2 has the following equivalent form.

(ii)' The principal solution of $(\mathrm{T})$ has no focal points in the interval $(0, N+1)_{\mathbb{Z}}$.

From Corollary 5.1 and Proposition 4.1(iii), we easily get the following.

Corollary 5.3 (Sturmian separation theorem). Assume that $S_{k}, T_{k}$ satisfy the conditions in Notation 2.1. If the principal solution of $(\mathrm{T})$ has a focal point in the interval $(0, N+1]_{\mathbb{Z}}$, then any other conjoined basis of $(\mathrm{T})$ has a focal point in $(0, N+1]_{\mathbb{Z}}$ as well.

The above result can be found as a special case of [23, Theorem 3] or [24, Corollary 3.1]. A refinement of the previous result can be deduced from [9, Theorem 1].

Corollary 5.4 (Sturmian separation theorem). Assume that $S_{k}, T_{k}$ satisfy the conditions in Notation 2.1. If there exists a conjoined basis of $(\mathrm{T})$ with no focal points in $(0, N+1]_{\mathbb{Z}}$, then any other conjoined basis of $(\mathrm{T})$ has at most $n$ focal points in $(0, N+1]_{\mathbb{Z}}$.

The corresponding proof of Corollary 5.4 in [9] is based on the construction of a suitable sequence $x=\left\{x_{k}\right\}_{k=0}^{N+1}$ with $x_{0}=0=x_{N+1}$ and $x \not \equiv 0$, for which the value of the functional $\mathfrak{f}_{T}(x)=0$, thus contradicting Proposition 4.1. Finally, the most general result in this direction is the following.

Theorem 5.5 (Sturmian separation theorem). Assume that $S_{k}, T_{k}$ satisfy the conditions in Notation 2.1. If the principal solution of $(\mathrm{T})$ has $m$ focal points in $(0, N+1]_{\mathbb{Z}}$, then any other conjoined basis of $(\mathrm{T})$ has at least $m$ and at most $m+n$ focal points in $(0, N+1]_{\mathbb{Z}}$.

Proof. This is a special case of $[8$, Theorem 3.1] for symplectic systems $(\mathbb{S})$, in which we use the coefficients from Corollary 3.2.

One can see that Corollary 5.4 is a special case of Theorem 5.5 for $m=0$.

By comparing the numbers of focal points in $(0, N+1]_{\mathbb{Z}}$ of two conjoined bases of $(\mathrm{T})$ with the number of focal points in $(0, N+1]_{\mathbb{Z}}$ of the principal solution of $(\mathrm{T})$, we obtain from Theorem 5.5 the classical Sturmian separation theorem; see [8, Theorem 1.1] and [25, page 366].

Corollary 5.6 (Sturmian separation theorem). Assume that $S_{k}, T_{k}$ satisfy the conditions in Notation 2.1. The difference between the numbers of focal points in $(0, N+1]_{\mathbb{Z}}$ of any two conjoined bases of $(\mathrm{T})$ is at most $n$.

The above Sturmian separation theorems are obtained from the comparison of the numbers of focal points in $(0, N+1]_{\mathbb{Z}}$ of conjoined bases of two (possibly) different recurrence equations $(\mathrm{T})$ or symplectic systems $(\mathbb{S})$. More precisely, in addition to $(\mathrm{T})$ consider the symmetric three-term recurrence equation

$$
\widetilde{S}_{k+1} x_{k+2}-\tilde{T}_{k+1} x_{k+1}+\tilde{S}_{k}^{T} x_{k}=0, \quad k \in[0, N-1]_{\mathbb{Z}}
$$


in which, as in Notation 2.1, the matrices $\widetilde{S}_{k}$ are invertible and $\widetilde{T}_{k}$ are symmetric. Following [23, Section 3.2] and [8, Theorem 1.2] we define the symmetric $2 n \times 2 n$ matrices

$$
\mathcal{G}_{k}:=\left(\begin{array}{cc}
T_{k} & -S_{k} \\
-S_{k}^{T} & 0
\end{array}\right), \quad \tilde{\mathcal{G}}_{k}:=\left(\begin{array}{cc}
\tilde{T}_{k} & -\widetilde{S}_{k} \\
-\widetilde{S}_{k}^{T} & 0
\end{array}\right)
$$

The following result from [1, Theorem 5.20] and [23, Theorem 4] is a comparison complement of the separation theorem in Corollary 5.3. It is a direct consequence of Proposition 4.1.

Corollary 5.7 (Sturmian comparison theorem). Assume that $S_{k}, T_{k}, \widetilde{S}_{k}$, and $\widetilde{T}_{k}$ satisfy the conditions in Notation 2.1. In addition, let

$$
\mathcal{G}_{k} \geq \tilde{\mathcal{G}}_{k} \quad \forall k \in[0, N]_{\mathbb{Z}}
$$

If the principal solution of $(\mathrm{T})$ has a focal point in $(0, N+1]_{\mathbb{Z}}$, then any conjoined basis of $(\widetilde{\mathrm{T}})$ has a focal point in $(0, N+1]_{\mathbb{Z}}$ as well.

More precise statements about the numbers of focal points of the principal solution of $(\widetilde{\mathrm{T}})$, respectively, of $(\mathrm{T})$, and the number of focal points of conjoined bases of $(\mathrm{T})$, respectively, of $(\tilde{T})$, are contained in the next two results. In particular, Corollary 5.7 is a special case of Theorem 5.8 for $m=0$.

Theorem 5.8 (Sturmian comparison theorem). Assume that $S_{k}, T_{k}, \widetilde{S}_{k}$, and $\widetilde{T}_{k}$ satisfy the conditions in Notation 2.1 and let condition (5.2) hold. If the principal solution of (T) has $m$ focal points in $(0, N+1]_{\mathbb{Z}}$, then any conjoined basis of $(\widetilde{\mathrm{T}})$ has at least $m$ focal points in $(0, N+1]_{\mathbb{Z}}$.

Proof. This result follows from [8, Theorem 1.3] for symplectic systems ( $\mathbb{S}$ ) with the coefficients from Corollary 3.2.

Theorem 5.9 (Sturmian comparison theorem). Assume that $S_{k}, T_{k}, \widetilde{S}_{k}$, and $\widetilde{T}_{k}$ satisfy the conditions in Notation 2.1 and let condition (5.2) hold. If the principal solution of $(\widetilde{\mathrm{T}})$ has $m$ focal points in $(0, N+1]_{\mathbb{Z}}$, then any conjoined basis of $(\mathrm{T})$ has at most $m+n$ focal points in $(0, N+1]_{\mathbb{Z}}$.

Proof. This result is a special case of [8, Theorem 1.2] for symplectic systems ( $\mathbb{S}$ ) with the coefficients from Corollary 3.2.

Remark 5.10. For the Jacobi difference equations, that is, for $(\mathrm{J})$ and another equation of the same type

$$
\Delta\left(\widetilde{R}_{k} \Delta x_{k}+\widetilde{Q}_{k}^{T} x_{k+1}\right)=\widetilde{P}_{k} x_{k+1}+\widetilde{Q}_{k} \Delta x_{k}, \quad k \in[0, N-1]_{\mathbb{Z}}
$$

where $\widetilde{P}_{k}, \widetilde{Q}_{k}, \widetilde{R}_{k}$ satisfy the conditions in Notation 2.2 , the matrices $\mathcal{G}_{k}$ and $\widetilde{\mathcal{G}}_{k}$ have the form

$$
\mathcal{G}_{k}=\left(\begin{array}{cc}
R_{k} & -S_{k} \\
-S_{k}^{T} & R_{k}+Q_{k}+Q_{k}^{T}+P_{k}
\end{array}\right), \quad \tilde{\mathcal{G}}_{k}=\left(\begin{array}{cc}
\widetilde{R}_{k} & -\widetilde{S}_{k} \\
-\widetilde{S}_{k}^{T} & \widetilde{R}_{k}+\widetilde{Q}_{k}+\widetilde{Q}_{k}^{T}+\widetilde{P}_{k}
\end{array}\right)
$$


(i) If $Q_{k}=\widetilde{Q}_{k} \equiv 0$ (so that $S_{k}=R_{k}$ and $\widetilde{S}_{k}=\widetilde{R}_{k}$ are symmetric), then the classical conditions $R_{k} \geq \widetilde{R}_{k}$ and $P_{k} \geq \widetilde{P}_{k}$ for all $k \in[0, N]_{\mathbb{Z}}$ are equivalent with condition (5.2). This can be seen from the calculation

$$
\left(\begin{array}{l}
c \\
d
\end{array}\right)^{T}\left(\mathcal{G}_{k}-\tilde{\mathcal{G}}_{k}\right)\left(\begin{array}{l}
c \\
d
\end{array}\right)=(c-d)^{T}\left(R_{k}-\widetilde{R}_{k}\right)(c-d)+d^{T}\left(P_{k}-\widetilde{P}_{k}\right) d \quad \text { for any } c, d \in \mathbb{R}^{n}
$$

(ii) If $R_{k} \equiv \widetilde{R}_{k}$ and $Q_{k} \equiv \widetilde{Q}_{k}$, then the condition $P_{k} \geq \widetilde{P}_{k}$ for all $k \in[0, N]_{\mathbb{Z}}$ is equivalent with condition (5.2).

\section{Applications in Eigenvalue Theory}

The Sturmian separation and comparison theorems, in particular Theorems 5.5, 5.8, and 5.9, are proven in [8] by using the Rayleigh principle and the oscillation theorem for symplectic systems, that is, a result connecting the number of focal points in $(0, N+1]_{\mathbb{Z}}$ of the principal solution of $(\mathbb{S})$ with the number of eigenvalues of an associated discrete symplectic eigenvalue problem. The applications of the theory of Section 3 to these results for the threeterm recurrence equations $(\mathrm{T})$ will be presented in this section.

Consider the eigenvalue problem for the symmetric three-term recurrence equation (T) of the form

$$
S_{k+1} x_{k+2}-T_{k+1} x_{k+1}+S_{k}^{T} x_{k}=-\lambda W_{k} x_{k+1}, \quad k \in[0, N-1]_{\mathbb{Z}}, x_{0}=0=x_{N+1},
$$

where the $n \times n$ matrices

$W_{k}$ are symmetric and positive definite for all $k \in[0, N]_{\mathbb{Z}}$.

We will denote the recurrence equation in $(E)$ by $\left(T_{\lambda}\right)$, so that $\left(T_{0}\right)=(T)$. By Corollary 3.2, equation $\left(T_{\curlywedge}\right)$ can be written as a discrete symplectic system

$$
x_{k+1}=\mathbb{A}_{k} x_{k}+\mathbb{B}_{k} u_{k}, \quad u_{k+1}=\mathbb{C}_{k} x_{k}+\mathbb{D}_{k} u_{k}-\lambda \mathbb{W}_{k} x_{k+1}, \quad k \in[0, N]_{\mathbb{Z}}, x_{0}=0=x_{N+1},
$$

with symmetric matrices $\mathbb{W}_{k}:=W_{k}$. Systems of the form (6.2) are known to be the right ones to study the "symplectic" eigenvalue problems; see [26, Remark 3(iii)]. Note that although the matrix $\mathbb{W}_{N}$ appears in the second equation of (6.2) for $k=N$, the value of $\mathbb{W}_{N}$ is irrelevant because it is multiplied by $x_{N+1}=0$.

Let $\widehat{X}(\lambda)=\left\{\widehat{X}_{k}(\lambda)\right\}_{k=0}^{N+1}$ be the principal solution of the recurrence equation $\left(\mathrm{T}_{\lambda}\right)$, that is, $\widehat{X}_{0}(\lambda) \equiv 0$ and $\widehat{X}_{1}(\lambda)=S_{0}^{-1}$ for all $\lambda \in \mathbb{R}$. This means that the initial conditions of the principal solution $\widehat{X}(\lambda)$ do not depend on $\lambda$. The principal solution, as well as other (matrix or vector) solutions of $\left(T_{\lambda}\right)$, depends in general on $\lambda$, so that we will emphasize this dependence in the notation of the solutions. A number $\lambda \in \mathbb{R}$ is an eigenvalue of the eigenvalue problem (E) if there exists a nontrivial solution $\left\{x_{k}(\lambda)\right\}_{k=0}^{N+1}$ of $(\mathrm{E})$, or equivalently, if $\operatorname{det} \widehat{X}_{N+1}(\lambda)=0$. In 
this case, the number $\operatorname{def} \widehat{X}_{N+1}^{T}(\lambda):=\operatorname{dim} \operatorname{Ker} \widehat{X}_{N+1}(\lambda)$ is the multiplicity of $\lambda$ as being an eigenvalue of (E). Denote by, including the multiplicities,

$$
\begin{gathered}
n_{1}(\lambda):=\text { the number of focal points of } \widehat{X}(\lambda) \text { in }(0, N+1]_{\mathbb{Z}} \\
n_{2}(\lambda):=\text { the number of eigenvalues of }(E) \text { which are less or equal to } \lambda .
\end{gathered}
$$

Then we have the following oscillation theorem for the three-term recurrence equations $(T)$.

Theorem 6.1 (Oscillation theorem). Assume that $S_{k}, T_{k}$ satisfy the conditions in Notation 2.1 and $W_{k}$ satisfies (6.1). Then for all $\lambda \in \mathbb{R}$, we have

$$
n_{1}(\lambda)=n_{2}(\lambda), \quad n_{1}(\lambda)=n_{1}(\lambda+), \quad n_{2}(\lambda)=n_{2}(\lambda+),
$$

where $n_{j}(\lambda+)$ for $j \in\{1,2\}$ denotes the right-hand limit of the function $n_{j}(\cdot)$ at $\lambda$.

Proof. We refer to [10, Theorem 2] in which we use the coefficients from Corollary 3.2. Note that the number $m$ in [10, Theorem 2] is here zero, because of our assumption (6.1) and the result of [8, Lemma 4.5].

Remark 6.2. Among other properties of the eigenvalue problem (E), we mention those which are characteristic for the self-adjoint systems. These results, obtained from the symplectic systems theory, can be found in [10, Proposition 2] and [8, Theorem 4.7]. For discrete Jacobi equations $(\mathrm{J})$ with $Q_{k} \equiv 0$, these properties are shown in [27, Theorem 4.1] or [28, Theorem 3.1].

(i) The eigenvalues of (E) are real and the eigenfunctions corresponding to different eigenvalues are orthogonal with respect to the inner product

$$
\langle x, y\rangle_{W}:=\sum_{k=0}^{N} x_{k+1}^{T} W_{k} y_{k+1}, \quad x=\left\{x_{k}\right\}_{k=0}^{N+1}, y=\left\{y_{k}\right\}_{k=0}^{N+1} .
$$

If $\langle x, y\rangle_{W}=0$, then we say that $x$ and $y$ are orthogonal and write $x \perp y$.

(ii) The total number of eigenvalues of (E) including their multiplicities is $r \leq n N$.

(iii) Every $x=\left\{x_{k}\right\}_{k=0}^{N+1}$ with $x_{0}=0=x_{N+1}$ can be expanded in terms of the (orthonormal system of) eigenfunctions $x^{[1]}, \ldots, x^{[r]}$, that is, $x=\sum_{i=1}^{r} c_{i} x^{[i]}$, where $c_{i}=\left\langle x, x^{[i]}\right\rangle_{W}$ for $i=1, \ldots, r$.

Our final application is the Rayleigh principle describing the variational properties of the eigenvalues of (E). Let $\lambda_{1} \leq \cdots \leq \lambda_{r}$ be the eigenvalues of (E), where each eigenvalue appears repeatedly according to its multiplicity, and let $x^{[1]}, \ldots, x^{[r]}$ be the corresponding orthonormal eigenfunctions, that is, $\left\langle x^{[i]}, x^{[j]}\right\rangle_{W}=\delta_{i j}$. We set $\lambda_{0}:=-\infty$ and $\lambda_{r+1}:=+\infty$. 
Theorem 6.3 (Rayleigh principle). Assume that $S_{k}, T_{k}$ satisfy the conditions in Notation 2.1 and $W_{k}$ satisfies (6.1). Then for every $m \in\{0, \ldots, r\}$ we have

$$
\lambda_{m+1}=\min \left\{\frac{\mathcal{F}_{T}(x)}{\langle x, x\rangle_{W}}, \text { where } x=\left\{x_{k}\right\}_{k=0}^{N+1} \not \equiv 0, x_{0}=0=x_{N+1}, x \perp x^{[1]}, \ldots, x^{[m]}\right\} .
$$

(If $m=0$, then the above orthogonality condition is empty.)

Proof. We refer to [8, Theorem 4.6] in which we use the coefficients from Corollary 3.2.

\section{Concluding Remarks}

In this section we make some final comments related to the topics of this paper.

Remark 7.1. In [7, Theorem 1(ix)], the notion of "no backward focal points" in $[k, k+1)_{\mathbb{Z}}$ was introduced for conjoined bases of discrete symplectic systems $(\mathbb{S})$. This yields another characterization of the positivity or nonnegativity of the functional $\mathcal{F}_{T}$ in terms of the nonexistence of these backward focal points in the interval $[0, N+1)_{\mathbb{Z}}$ or in the interval $(0, N+1)_{\mathbb{Z}}$ for the principal solution $\bar{X}$ of $(\mathrm{T})$ at $N+1$. This principal solution is given by the initial conditions $\bar{X}_{N+1}=0$ and $\bar{X}_{N}=S_{N}^{T-1}$. In this respect, the results presented in Sections 5 and 6 can be transformed into this theory of backward focal points. However, this direction will not be pursued further on in this paper. Some recent results for symplectic systems $(\mathbb{S})$ related to this notion of backward focal points can be found in [11-13,29].

Remark 7.2. Based on the equivalence of the symmetric three-term recurrence equation (T) and Jacobi equation (J) in Proposition 2.4 and Corollary 3.3, all the results presented in Sections 4-6 for equations (T) remain valid also for Jacobi equations ( $\mathrm{J}$ ), for which the invertibility of $S_{k}:=R_{k}+Q_{k}^{T}$ is assumed (as in Notation 2.2), while the invertibility of $R_{k}$ is not imposed.

Remark 7.3. In [6, Corollaries 5.11 and 5.12], we showed the equivalence of the Jacobi equation (J) and the Jacobi equation

$$
\Delta\left[\left(\underline{R}_{k}-\underline{Q}_{k}\right) \Delta x_{k}+\left(\underline{Q}_{k}^{T}-\underline{P}_{k}\right) x_{k}\right]=\underline{P}_{k} x_{k}+\underline{Q}_{k} \Delta x_{k}, k \in[0, N-1]_{\mathbb{Z}}
$$

Both Jacobi equations $(\mathrm{J})$ and $(\mathrm{J})$ arise in the discrete calculus of variations problems- $(\mathrm{J})$ in problems with shift $x_{k+1}$ in the state variable, while (J) in problems with no shift $x_{k}$ in the state variable; see [30]. Therefore, all the results in this paper are valid also for the Jacobi equations of the type (J). In this setting, it is assumed that the matrices $\underline{S}_{k}:=\underline{R}_{k}-\underline{Q}_{k}$ are invertible.

For completeness, the transformations between the Jacobi equations $(\mathrm{J})$ and $(\mathrm{J})$ are displayed in Corollaries 7.5 and 7.6 below. For details see [6, Corollaries 5.11 and 5.12].

Notation 7.4 (Jacobi equation (J)). The matrices $\underline{P}_{k}, \underline{Q}_{k^{\prime}} \underline{R}_{k}$, and the vectors $x_{k}$ in (J) have the following properties: $\underline{P}_{k}, \underline{Q}_{k^{\prime}} \underline{\underline{R}}_{k} \in \mathbb{R}^{n \times n}$ are defined on $[0, N]_{\mathbb{Z}}, \underline{P}_{k}$ and $\underline{R}_{k}$ are symmetric, and the matrix $\underline{S}_{k}:=\underline{R}_{k}-\underline{Q}_{k}$ is invertible; $x_{k} \in \mathbb{R}^{n}$ are defined on $[0, N+1]_{\mathbb{Z}}$. 
Corollary 7.5 (Jacobi (J) to Jacobi (J)). Assume that $\underline{P}_{k^{\prime}} \underline{Q}_{k^{\prime}} \underline{R}_{k^{\prime}}$ and $\underline{S}_{k}$ satisfy the conditions in Notation 7.4. Then the Jacobi equation (J) is the Jacobi equation (J), whose coefficients

$$
P_{k}:=\underline{P}_{k}, \quad Q_{k}:=\underline{Q}_{k}-\underline{P}_{k}, \quad R_{k}:=\underline{R}_{k}-\underline{Q}_{k}-\underline{Q}_{k}^{T}+\underline{P}_{k}
$$

with $S_{k}=\underline{S}_{k}$ satisfy the conditions in Notation 2.2.

Corollary 7.6 (Jacobi (J) to Jacobi (J)). Assume that $P_{k}, Q_{k}, R_{k}$, and $S_{k}$ satisfy the conditions in Notation 2.2. Then the Jacobi equation $\left.{ }^{-} \mathrm{J}\right)$ is the Jacobi equation (J), whose coefficients

$$
\underline{P}_{k}:=P_{k}, \quad \underline{Q}_{k}:=P_{k}+Q_{k}, \quad \underline{R}_{k}:=R_{k}+Q_{k}+Q_{k}^{T}+P_{k}
$$

with $\underline{S}_{k}=S_{k}$ satisfy the conditions in Notation 7.4.

\section{Acknowledgments}

This research supported by the Czech Science Foundation under Grant 201/10/1032, and by the research projects MSM 0021622409 and ME 891 (program Kontakt) of the Ministry of Education, Youth, and Sports of the Czech Republic. It was also supported by the National Science Foundation under Grant DMS - 0707789.

\section{References}

[1] C. D. Ahlbrandt and A. C. Peterson, Discrete Hamiltonian Systems: Difference Equations, Continued Fractions, and Riccati Equations, vol. 16 of Kluwer Texts in the Mathematical Sciences, Kluwer Academic, Boston, Mass, USA, 1996.

[2] C. D. Ahlbrandt, "Discrete variational inequalities," in "General Inequalities 6", Proceedings of the 6th International Conference on General Inequalities (Oberwolfach, 1990), W. Walter, Ed., vol. 103 of International Series of Numerical Mathematics, pp. 93-107, Birkhäuser, Basel, Switzerland, 1992.

[3] R. Hilscher and V. Zeidan, "Coupled intervals in the discrete calculus of variations: necessity and sufficiency," Journal of Mathematical Analysis and Applications, vol. 276, no. 1, pp. 396-421, 2002.

[4] R. Hilscher and V. Zeidan, "Nonnegativity of a discrete quadratic functional in terms of the (strengthened) Legendre and Jacobi conditions," Computers E Mathematics with Applications, vol. 45, no. 6-9, pp. 1369-1383, 2003.

[5] R. Hilscher and V. Zeidan, "Nonnegativity and positivity of quadratic functionals in discrete calculus of variations: a survey," Journal of Difference Equations and Applications, vol. 11, no. 9, pp. 857-875, 2005.

[6] R. Šimon Hilscher and V. Zeidan, "Symplectic structure of Jacobi systems on time scales," International Journal of Difference Equations, vol. 5, no. 1, pp. 55-81, 2010.

[7] M. Bohner and O. Došlý, "Disconjugacy and transformations for symplectic systems," The Rocky Mountain Journal of Mathematics, vol. 27, no. 3, pp. 707-743, 1997.

[8] M. Bohner, O. Došlý, and W. Kratz, "Sturmian and spectral theory for discrete symplectic systems," Transactions of the American Mathematical Society, vol. 361, no. 6, pp. 3109-3123, 2009.

[9] O. Došlý and W. Kratz, "A Sturmian separation theorem for symplectic difference systems," Journal of Mathematical Analysis and Applications, vol. 325, no. 1, pp. 333-341, 2007.

[10] O. Došlý and W. Kratz, "Oscillation theorems for symplectic difference systems," Journal of Difference Equations and Applications, vol. 13, no. 7, pp. 585-605, 2007.

[11] O. Došlý and W. Kratz, "A remark on focal points of recessive solutions of discrete symplectic systems," Journal of Mathematical Analysis and Applications, vol. 363, no. 1, pp. 209-213, 2010.

[12] J. V. Elyseeva, "Transformations and the number of focal points for conjoined bases of symplectic difference systems," Journal of Difference Equations and Applications, vol. 15, no. 11-12, pp. 1055-1066, 2009. 
[13] J. V. Elyseeva, "Comparative index for solutions of symplectic difference systems," Differential Equations, vol. 45, no. 3, pp. 445-459, 2009, translated from: Differencial'nyje Uravnenija 45 (2009), no. 3, 431-444.

[14] R. Hilscher and V. Růžičková, "Riccati inequality and other results for discrete symplectic systems," Journal of Mathematical Analysis and Applications, vol. 322, no. 2, pp. 1083-1098, 2006.

[15] R. Hilscher and V. Růžičková, "Implicit Riccati equations and quadratic functionals for discrete symplectic systems," International Journal of Difference Equations, vol. 1, no. 1, pp. 135-154, 2006.

[16] R. Hilscher and V. Zeidan, "Symplectic difference systems: variable stepsize discretization and discrete quadratic functionals," Linear Algebra and its Applications, vol. 367, pp. 67-104, 2003.

[17] R. Hilscher and V. Zeidan, "Coupled intervals for discrete symplectic systems," Linear Algebra and its Applications, vol. 419, no. 2-3, pp. 750-764, 2006.

[18] W. Kratz, "Discrete oscillation," Journal of Difference Equations and Applications, vol. 9, no. 1, pp. 135$147,2003$.

[19] R. Hilscher and V. Zeidan, "Extension of discrete LQR-problem to symplectic systems," International Journal of Difference Equations, vol. 2, no. 2, pp. 197-208, 2007.

[20] C. D. Ahlbrandt and M. Heifetz, "Discrete Riccati equations of filtering and control," in Proceedings of the 1st International Conference on Difference Equations (San Antonio, Texas, 1994), S. Elaydi, J. Graef, G. Ladas, and A. Peterson, Eds., pp. 1-16, Gordon and Breach, Newark, NJ, USA.

[21] W. T. Reid, Riccati Differential Equations, Academic Press, New York, NY, USA, 1972.

[22] R. Hilscher and V. Zeidan, "Riccati equations for abnormal time scale quadratic functionals," Journal of Differential Equations, vol. 244, no. 6, pp. 1410-1447, 2008.

[23] R. Hilscher, "Reid roundabout theorem for symplectic dynamic systems on time scales," Applied Mathematics and Optimization, vol. 43, no. 2, pp. 129-146, 2001.

[24] R. Hilscher and V. Zeidan, "Applications of time scale symplectic systems without normality," Journal of Mathematical Analysis and Applications, vol. 340, no. 1, pp. 451-465, 2008.

[25] W. T. Reid, Ordinary Differential Equations, John Wiley \& Sons, New York, NY, USA, 1971.

[26] M. Bohner, O. Došlý, and W. Kratz, "An oscillation theorem for discrete eigenvalue problems," The Rocky Mountain Journal of Mathematics, vol. 33, no. 4, pp. 1233-1260, 2003.

[27] Y. Shi and S. Chen, "Spectral theory of second-order vector difference equations," Journal of Mathematical Analysis and Applications, vol. 239, no. 2, pp. 195-212, 1999.

[28] G. Shi and H. Wu, "Spectral theory of Sturm-Liouville difference operators," Linear Algebra and Its Applications, vol. 430, no. 2-3, pp. 830-846, 2009.

[29] O. Došlý, "Oscillation theory of symplectic difference systems," in "Advances in Discrete Dynamical Systems", Proceedings of the 11th International Conference on Difference Equations and Applications (Kyoto, 2006), S. Elaydi, K. Nishimura, M. Shishikura, and N. Tose, Eds., vol. 53 of Advanced Studies in Pure Mathematics, pp. 41-50, Mathematical Society of Japan, Tokyo, Japan, 2009.

[30] R. Šimon Hilscher, "A note on the time scale calculus of variations problems," in Ulmer Seminare über Funktionalanalysis und Differentialgleichungen, vol. 14, pp. 223-230, University of Ulm, Ulm, Germany, 2009. 